\title{
In Vitro Synergistic Antimicrobial Activity of Combinations of Meropenem, Colistin, Tigecycline, Rifampin, and Ceftolozane/Tazobactam Against Carbapenem-Resistant Acinetobacter Baumannii
}

\author{
Yong Guk Ju \\ Korea University College of Medicine \\ Hak Joon Lee \\ Korea University College of Medicine \\ Hong Soon Yim \\ Korea University College of Medicine \\ Chang Kyu Lee \\ Korea University College of Medicine \\ Mingoo Lee \\ Korea University College of Medicine \\ Jang Wook Sohn \\ Korea University College of Medicine \\ Young Kyung Yoon ( $\nabla$ young7912@korea.ac.kr) \\ Korea University College of Medicine
}

\section{Research Article}

Keywords: Acinetobacter baumannii, combination therapy, antibiotic synergism

Posted Date: December 28th, 2021

DOI: https://doi.org/10.21203/rs.3.rs-1168856/v1

License: (c) (1) This work is licensed under a Creative Commons Attribution 4.0 International License. Read Full License 


\section{Abstract}

The aim of this study was to investigate the in vitro activity of various antimicrobial combinations against carbapenem-resistant Acinetobacter baumannii (CRAB) isolates producing OXA-23 carbapenemases.

In vitro activity of six two-drug combinations against CRAB isolates collected from patients with CRAB bacteremia was evaluated using the checkerboard method and time-kill assay [0.5 $\times, 1 \times, 2 \times$ minimum inhibitory concentrations (MIC)], to identify potential synergistic and bactericidal two-drug combinations against CRAB isolates, using meropenem, colistin, tigecycline, rifampin, and ceftolozane/tazobactam. All 10 CRAB isolates in our study carried the OXA-58type and OXA-23-type carbapenem-hydrolyzing oxacillinase. The colistin-ceftolozane/tazobactam combination demonstrated a synergistic effect in both the time-kill assay (using an antibiotic concentration of $1 \times \mathrm{MIC}$ ) and the checkerboard method, while simultaneously showing a bactericidal effect in the time-kill assay. For all $10 \mathrm{CRAB}$ isolates, time-kill curves showed a significant synergistic bactericidal activity of the colistin-ceftolozane/tazobactam combination at $0.5 \times$ MIC. Overall, there is substantial discordance of synergistic activity between the checkerboard microdilution and time-kill assay (with a concordance of $35 \%$ ). Our study demonstrated that the two-drug combinations of colistin and ceftolozane/tazobactam can be a potential alternative for treating CRAB infections. The effect of these antibiotic combinations should be evaluated through clinical trials.

\section{Introduction}

Carbapenem-resistant Acinetobacter baumannii (CRAB), a leading nosocomial pathogen, poses a global threat to public health. ${ }^{1}$ This pathogen is resistant to most of the available antibiotics in clinical practice and has extremely limited treatment options with a consequent increase in mortality. ${ }^{2-4}$ Furthermore, the spread of CRAB in a hospital environment is a thorny problem for infection control. They can colonize various body parts of hospitalized patients and survive for a long time on the surface of the hospital facilities. ${ }^{5,6}$

In the Republic of Korea, the carbapenem resistance rate of $A$. baumannii isolated from patients hospitalized at intensive care units was $90 \%{ }^{7,8}$ According to the Korean part of the Global Antimicrobial Resistance Surveillance System, CRAB is the most common multidrug-resistant pathogen causing bloodstream infection at intensive care units, with an incidence of 6.3 cases per 10,000 patient-days. ${ }^{9}$

The World Health Organization has ranked CRAB as a pathogen of critical priority in the global priority list of multidrug-resistant bacteria urging the development of new antibiotics. ${ }^{10}$ Despite the relentless attempts to improve therapeutic approaches, there is no promising new antibiotic that can convincingly control CRAB infections. ${ }^{11}$ Currently, only a few antibiotics of uncertain efficacy, such as colistin and tigecycline, are available for treating CRAB infections. The reduced susceptibility of CRAB, unfavorable pharmacokinetic properties, unclear optimal dosing, and potential adverse effects are barriers to the clinical use of existing drugs such as colistin and tigecycline.

Given the increasing multidrug-resistance rates and lack of effective antibiotics, combination therapy can be considered as an alternative interim strategy for effective management of CRAB infections. Antimicrobial combination therapy may broaden the spectrum of activity, minimize the development of antimicrobial resistance, and synergistically inactivate microorganisms. Several mechanisms proposed for synergistic antibacterial effects include enhanced bioavailability, inhibitor inhibition, sequential blockade, mutual stabilization, parallel pathway inhibition, and regulation modulation. ${ }^{12}$ However, no standardized method has been established for the in vitro evaluation of combination therapies by the Clinical and Laboratory Standards Institute (CLSI). ${ }^{13-15}$ Nevertheless, several studies have addressed the therapeutic potential of combination therapy against CRAB infections. ${ }^{16-20}$

The purpose of this study was to investigate the in vitro activity of antimicrobial combinations of meropenem, colistin, tigecycline, rifampin, and ceftolozane/tazobactam against CRAB isolates producing OXA-23 carbapenemases.

\section{Methods}

\section{Study population}

A total of 158 clinical isolates of $A$. baumannii were collected from nonduplicate patients with CRAB bacteremia in a 1,048-bed tertiary care hospital in Seoul, Republic of Korea from April, 2018 to January, 2020. Finally, 10 clinical isolates of CRAB exhibiting resistance to imipenem, meropenem, and ertapenem were randomly selected. ${ }^{21}$

The study protocol was approved prior to study initiation by the Institutional Review Board of Korea University Anam Hospital [No. 2020AN0157]. The study were performed in accordance with the ethical principles outlined in the Declaration of Helsinki. Informed consent was obtained from all subjects involved in this study.

\section{Bacterial isolates and antimicrobial susceptibility testing}

The identification and antimicrobial susceptibility testing of $A$. baumannii strains were performed initially using the MicroScan Pos Combo Panel Type 6 automated system (Baxter Diagnostics, West Sacramento, CA, USA) in a clinical microbiology laboratory. Confirmation of the identification of $A$. baumannii strains was performed using a matrix-assisted laser desorption/ionization-time of flight mass spectrometry (Bruker Daltonics, Bremen, Germany).

The minimum inhibitory concentration (MIC) was determined for each of the following 15 antimicrobial agents using the broth microdilution method, with the Cation-Adjusted Mueller-Hinton $₫$ broth (CA-MHB) (Becton Dicknson \& Co., Sparks, MD, USA) and geometric 2-fold serial dilutions according to the CLSI recommendations: ${ }^{21,22}$ meropenem (Yuhan Co, Seoul, Korea), ertapenem (MSD-Chibret, France), colistin (SteriMax Inc., Ont, Canada), amikacin (shinpoong Co, 
Seoul, Korea), tigecycline (Pfizer Pharmaceutical, Seoul, Korea), ceftolozane/tazobactam (Wyeth; Madison, NJ, USA), piperacillin/tazobactam (Chong kun dang pharmaceutical Co, Seoul, Korea)), ampicillin/sulbactam (Keun-hwa pharm, Seoul, Korea), ceftazidime (Hanmi Co, Seoul, Korea), cefepime (Boryung Co, Seoul, Korea), astreonam (Crystal lifesciences, Cheonju, Korea), minocycline (Skchemical Co, Seongnam, Korea), fosfomycin (Pharmbio Co, Chungju, Korea), rifampin (Yuhan Co, Seoul, Korea), and ciprofloxacin (Bayer AG, Leverkusen, Germany). The MIC results were interpreted according to the CLSI breakpoint criteria. ${ }^{21}$ No breakpoints are available for tigecycline from the CLSI guidelines. ${ }^{21}$ Therefore, the criteria of the United States Food and Drug Administration for Enterobacteriaceae were used for tigecycline (susceptibility, $2 \mathrm{mg} / \mathrm{L}$; resistance, $8 \mathrm{mg} / \mathrm{L}$ ). ${ }^{23}$ For interpretation of colistin and rifampin susceptibilities, the breakpoints proposed by Gales et al. (resistance, $4 \mathrm{mg} / \mathrm{L}$ ) and Hogg et al. (resistance, $2 \mathrm{mg} / \mathrm{L}$ ) were used respectively. ${ }^{24,25}$ Pseudomonas aeruginosa (ATCC 27853) was used as a quality control isolate.

All CRAB isolates from blood cultures were immediately stored in Brain Heart Infusion broth (Becton Dickinson \& Co., Sparks, MD, USA) containing 20\% glycerol and kept in a freezer at $-70{ }^{\circ} \mathrm{C}$ until February 2020 . The isolates were thawed, and primary and secondary cultures were inoculated in $5 \%$ sheep blood agar for the experiments.

\section{Detection of carbapenem resistance determinants}

For all CRAB isolates, Ambler class B metallo- $\beta$-lactamase genes ( $b / a_{\mathrm{IMP}-1}, b / a_{\mathrm{IMP}-2}, b / a_{\mathrm{VIM}-1}$, and $\left.b \mathrm{a}_{\mathrm{VIM}-2}\right)$ and Ambler class D OXA-type carbapenemasesencoding genes ( $b / a_{\text {oxa-23 }}, b / a_{\text {oxa-24, }}$ bla oxa-51, and $\left.b / a_{\text {oxa-58 }}\right)$ were detected using the polymerase chain reaction (PCR) method or multiplex PCR technique. The primers used in this study are shown in Table 1.

Each reaction mixture $(20 \mu \mathrm{L})$ contained $1 \mu \mathrm{L}$ of genomic DNA, 10 pmol of each primer, $1 \mathrm{U}$ of Taq DNA polymerase, $0.25 \mathrm{mM} \mathrm{dNTP}, 10 \mathrm{mM}$ Tris-HCl (pH 9.0), $40 \mathrm{mM} \mathrm{KCl}$, and $1.5 \mathrm{mM} \mathrm{MgCl}_{2}$. PCR reaction temperatures for the Ambler class B metallo- $\beta$-lactamase genes were $94^{\circ} \mathrm{C}$ for $5 \mathrm{~min}$, followed by 30 cycles of $94{ }^{\circ} \mathrm{C}$ for $45 \mathrm{~s}$, at each specific annealing temperature for $1 \mathrm{~min}$ and $72{ }^{\circ} \mathrm{C}$ for $1 \mathrm{~min}$, followed by a final extension at $72{ }^{\circ} \mathrm{C}$ for $7 \mathrm{~min}(\mathrm{Table} 1)$. PCR reaction temperatures for the Ambler class D OXA-type carbapenemase-encoding genes were $94{ }^{\circ} \mathrm{C}$ for 5 min, followed by 30 cycles of $94{ }^{\circ} \mathrm{C}$ for $30 \mathrm{~s}$, at each specific annealing temperature for $40 \mathrm{~s}$ and $72{ }^{\circ} \mathrm{C}$ for $50 \mathrm{~s}$, followed by a final extension at $72{ }^{\circ} \mathrm{C}$ for $7 \mathrm{~min}$ (Table 1).

\section{Checkerboard assays for synergy testing}

The synergistic activities of various two-drug combinations against the 10 CRAB isolates were evaluated using the checkerboard assay, which was conducted in 96-well microtiter plates (Corning Inc., Kennebunk, ME, USA). In brief, panels of 96-well microtiter plates were prepared according to results obtained from the MIC of each antibiotic determined with broth microdilution. Dilution intervals were determined from 2-32 times higher and 1/8-1/64 below the MIC values obtained from the preliminary analysis. The antibiotic stock solutions were diluted with CA-MHB, and the concentrations of the upper left part of the plates were set to 0 . The rows of the plates contained $50 \mu \mathrm{L}$ of two-fold serial dilutions of the first antibiotic in each well, and the columns contained $50 \mu \mathrm{L}$ of twofold serial dilutions of the second antibiotic. The ranges of test concentrations of each antibiotic in combinations were as follows: colistin, $0-128 \mathrm{mg} / \mathrm{L}$; meropenem, 0-128 mg/L; tigecycline, 0-8 mg/L; ceftolozane/tazobactam, 0-128 mg/L; and rifampin, 0-256 mg/L.

The A. baumannii inocula consisted of $100 \mu \mathrm{L}$ of a two-fold dilution of a $0.5 \mathrm{McFarland}$ turbidity standard prepared in CA-MHB. The final inoculum concentration was $5 \times 10^{4} \mathrm{CFU} / \mathrm{mL}$ in each well. Except for the sterility control well, all the wells were inoculated and then incubated at $37^{\circ} \mathrm{C}$ for $18-20 \mathrm{~h}$, and then the wells were diluted to an OD $600 \mathrm{~nm}$ measured at the stationary phase with an absorbance microplate reader (SpectraMax Plus 384 , Molecular Devices, Inc). The MIC was determined as the well in the microtiter plate with the lowest drug concentration at which there was no visible growth. The fractional inhibitory concentration index (FICl) was calculated using the formula below.

$\mathrm{FICl}=[(\mathrm{MIC}$ of drug A in combination $) /(\mathrm{MIC}$ of drug A alone $)]+[(\mathrm{MIC}$ of drug B in combination $) /(\mathrm{MIC}$ of drug B alone $)]$

Interpretation of the $\mathrm{FICl}$ was as follows: $\mathrm{FICl} \leq 0.5$, synergistic; $0.5<\mathrm{FICl} \leq 1$, additive; $1<\mathrm{FICl} \leq 4$, indifferent; and $\mathrm{FICl}>4$, antagonistic. ${ }^{26}$

\section{Time-kill assay for synergy testing}

Along with the checkerboard assay, time-kill assays were conducted on the $10 \mathrm{~A}$. baumannii isolates. In brief, tubes containing freshly prepared CA-MH broth supplemented with the antibiotics, alone and in combination, were inoculated with CRAB isolates at a concentration of $10^{4} \mathrm{CFU} / \mathrm{mL}$. The tubes had a final volume of $10 \mathrm{~mL}$ and were incubated at $37^{\circ} \mathrm{C}$ in a shaking incubator $(200 \mathrm{rpm})$, in ambient air.

Then, $100 \mu \mathrm{L}$ aliquots were obtained from each tube at $0,2,4,8,12$, and $24 \mathrm{~h}$ of incubation and serially diluted in saline for determination of viable counts. Diluted samples $(10 \mu \mathrm{L})$ were plated on CA-MHA plates using a spreader (SPL life Science, Co) and incubated at $37^{\circ} \mathrm{C}$ for $18-24 \mathrm{~h}$, and then the number of colonies formed was counted. The antibiotic carry-over effect was minimized by washing the aliquots in sterile phosphate-buffered saline (PBS) and centrifuging for $5 \mathrm{~min}$ at 1,300 rpm before a 10 -fold serial dilution in sterile PBS. The initial bacterial density from the original sample was calculated based on the dilution factor. The lower limit of detection for the colony counts was $2 \log _{10} \mathrm{CFU} / \mathrm{mL}$. The concentrations of antibiotics applied were $0.5 \times \mathrm{MIC}, 1 \times \mathrm{MIC}$, and $2 \times$ MIC alone or in combination.

The bactericidal activity of single antibiotics or combinations was defined as a decrease of $\geq 4 \log _{10}$ in $24 \mathrm{~h}$ compared with the number of viable cells at the initial time point. ${ }^{27}$ A synergistic effect was considered as a decrease of $\geq 2 \log _{10} \mathrm{CFU} / \mathrm{mL}$ in 6 or $24 \mathrm{~h}$ when comparing the antibiotics in combination with the most active drug alone at the different time points, whilst an increase of $>2 \log _{10}$ was indicative of antagonism. Indifference was considered as any other outcome that did not meet the criteria for either synergy or antagonism. ${ }^{28}$ 


\section{Results}

\section{Characteristics of $A$. baumannii clinical isolates}

Clinical isolates involved in this study are listed in Table 2. Out of 10 patients with CRAB bacteremia, $50 \%$ had in-hospital mortality. All 10 CRAB isolates carried the OXA-58-type and OXA-23-type carbapenem-hydrolyzing oxacillinase, whereas they did not harbor class B metallo-carbapenemases or other class D carbapenemases. Ten clinical isolates were resistant to meropenem with MICs of $64 \mathrm{mg} / \mathrm{L}$. Among them, the susceptibility rate to each antibiotic is as follows: tigecycline $90 \%$, minocycline $100 \%$, rifampin $30 \%$, colistin $10 \%$, ceftolozane/tazobactam $0 \%$, ciprofloxacin $0 \%$, fosfomycin $0 \%$, aztreonam $0 \%$, ceftazidime $0 \%$, ampicillin/sulbactam $0 \%$, piperacillin/tazobactam $0 \%$, and amikacin $0 \%$ (Table 2).

\section{Checkerboard assay against $A$. baumannii clinical isolates}

The results of the in vitro synergism and MIC values of the individual antibiotics in combination against the 10 CRAB isolates using the checkerboard assay are summarized in Table 3. In terms of checkerboard assay, in vitro synergistic activities $\left(\sum \mathrm{FICl} \leq 0.5\right)$ against CRAB isolates were most frequently observed for meropenem-tigecycline combination (90\%), followed by ceftolozane/tazobactam-meropenem combination (70\%), meropenem-rifampin combination (70\%), ceftolozane/tazobactam-colistin combination (60\%), colistin-tigecycline combination (30\%), and meropenem-colistin combination (30\%) (Table 3). All combinations displayed synergism to a certain extent.

Notably, antagonistic interactions were not observed in our study. The MICs of the antibiotics in combination were lower than the MICs of the antibiotics used as a single agent.

\section{Time-kill assay against $A$. baumannii clinical isolates}

All 10 CRAB isolates were evaluated using the time-kill method. When using an antibiotic concentration of $0.5 \times \mathrm{MIC}, 1 \times \mathrm{MIC}$, and $2 \times \mathrm{MIC}$, bactericidal activity of antibiotic monotherapy on the 10 CRAB isolates was found in meropenem ( $0.5 \times \mathrm{MIC}, 0 \%, 0 / 10 ; 1 \times \mathrm{MIC}, 40 \%, 4 / 10 ; 2 \times \mathrm{MIC}, 70 \%, 7 / 10)$, ceftolozane/tazobactam (0.5 $\mathrm{MIC}, 0 \%, 0 / 10 ; 1 \times \mathrm{MIC}, 10 \%, 1 / 10 ; 2 \times \mathrm{MIC}, 70 \%, 7 / 10)$, colistin (0.5× MIC, 0\%, 0/10; $1 \times \mathrm{MIC}, 50 \%, 5 / 10 ; 2 \times \mathrm{MIC}, 70 \%, 7 / 10)$, rifampin (0.5× MIC, $0 \%, 0 / 10 ; 1 \times$ MIC, $10 \%, 1 / 10 ; 2 \times$ MIC, 40\%, 4/10) and tigecycline $(0.5 \times \mathrm{MIC}, 10 \%, 1 / 10 ; 1 \times \mathrm{MIC}, 10 \%, 1 / 10 ; 2 \times \mathrm{MIC}, 40 \%, 4 / 10)$ within $24 \mathrm{~h}$ (Supplementary file).

For the time-kill assay, using an antibiotic concentration of $1 \times \mathrm{MIC}$, the in vitro synergistic activities against the 10 CRAB isolates were most frequently observed for the meropenem-colistin combination (100\%), ceftolozane/tazobactam-colistin (100\%), and tigecycline-colistin combination (100\%), followed by the meropenem-tigecycline combination (70\%), ceftolozane/tazobactam-meropenem combination (60\%), and meropenem-rifampin combination (30\%) within $24 \mathrm{~h}$ (Table 4).

For the colistin-ceftolozane/tazobactam combination (both at a concentration of $1 \times \mathrm{MIC}$ ), the $10 \mathrm{CRAB}$ isolates yielded synergy rates of $60 \%$ in $12 \mathrm{~h}$ and $50 \%$ in $24 \mathrm{~h}$, respectively (Figure 1). For the colistin-meropenem combination (at $1 \times \mathrm{MIC}$ ), the $10 \mathrm{CRAB}$ isolates showed synergistic rates of $50 \%$ in $12 \mathrm{~h}$ and $40 \%$ in $24 \mathrm{~h}$, respectively (Figure 1). For the meropenem-ceftolozane/tazobactam combination (at $1 \times \mathrm{MIC}$ ), the 10 CRAB isolates showed synergistic rates of $20 \%$ in $12 \mathrm{~h}$ and $50 \%$ in $24 \mathrm{~h}$, respectively (Figure 1 ). For the meropenem-tigecycline combination (at $1 \times \mathrm{MIC}$ ), the 10 CRAB isolates showed synergistic rates of $40 \%$ in $12 \mathrm{~h}$ and $50 \%$ in $24 \mathrm{~h}$, respectively (Figure 1). However, the meropenem-rifampin and colistin-tigecycline combinations (at $1 \times$ MIC) did not show synergy rates of more than $50 \%$ (Figure 1). Overall, for six combinations of antibiotics, doubling the antibiotic concentration did not improve synergy rates (Figure 1 ). Unlike synergistic inhibitory activity for other antibiotic combinations sustained until $24 \mathrm{~h}$, the CRAB 34 isolates demonstrated a regrowth at $4 \mathrm{~h}$ for the colistin-ceftolozane/tazobactam combination (both at a concentration of $1 \times \mathrm{MIC}$ ) (Figure 2).

For the time-kill assay, using an antibiotic concentration of $1 \times \mathrm{MIC}$, in vitro bactericidal activities for the 10 CRAB isolates were most frequently observed for the meropenem-colistin combination (100\%), ceftolozane/tazobactam-colistin combination (100\%), and tigecycline- colistin combination (100\%), followed by the meropenem-tigecycline combination (50\%), ceftolozane/tazobactam-meropenem combination (50\%), and meropenem-rifampin combination (30\%) within $24 \mathrm{~h}$ (Table 4).

At $1 \times$ and $2 \times$ MIC concentrations, the combinations of colistin-ceftolozane/tazobactam $(1 \times$ MIC, $100 \%, 10 / 10 ; 2 \times$ MIC, 100\%, $10 / 10)$, colistin-tigecycline (1 $\times$ MIC, 70\%, 7/10; $2 \times$ MIC, 100\%, 10/10), and colistin-meropenem (1 $\times$ MIC, 100\%, 10/10; $2 \times$ MIC, 100\%, 10/10) against the 10 CRAB isolates, showed bactericidal activity usually beginning at $12 \mathrm{~h}$ after inoculation (Figure 1 ).

With respect to the time-kill assay test, at $1 \times$ MIC concentrations, antibiotic combinations that showed both bactericidal activity and synergistic effect within $24 \mathrm{~h}$ are as follows: colistin-ceftolozane/tazobactam (70\%), colistin-tigecycline (70\%), colistin-meropenem (50\%), meropenem-ceftolozane/tazobactam (50\%), meropenem-tigecycline (30\%), and rifampin-meropenem (30\%) (Table 5). However, the frequency of simultaneously showing both effects at the same timepoint was relatively low (Figure 1). In more than $50 \%$ of the 10 clinical isolates, antibiotic combinations showing both bactericidal activity and synergistic effect at the same time-point, were colistin-ceftolozane/tazobactam (60\% and $50 \%$ in $12 \mathrm{~h}$ and $24 \mathrm{~h}$, respectively, after inoculation) and colistin-meropenem (50\% at $12 \mathrm{~h}$ after inoculation) (Figure 1$)$.

Regarding the synergistic effect of antibiotic combinations, 35\% (21/60) of the results of the time-kill assay were consistent with the checkerboard results (Table 5). Both the time-kill assay and the checkerboard assay showed synergistic effects, while simultaneously showing bactericidal effects in the time-kill assay as follows: colistin-ceftolozane/tazobactam (40\%), meropenem-tigecycline (30\%), meropenem-ceftolozane/tazobactam (30\%), colistin-meropenem (30\%), colistin-tigecycline (20\%), and rifampin-meropenem (20\%) within $24 \mathrm{~h}$ (Table 5). 
The time-kill curves of six different combinations of antibiotics with $0.5 \times, 1 \times, 2 \times$ MIC concentrations against the CRAB isolates are shown in Figure 3 . No antagonism was observed in the time-kill assay for the antibiotic combinations (Supplementary file).

\section{Discussion}

Our study was performed to determine which antibiotic combinations might be potentially suitable options for treating CRAB infections. This is the first study, to the best of our knowledge, to evaluate the in vitro synergistic activity of ceftolozane/tazobactam with other antibiotics against CRAB isolates. Our findings revealed that the combination of colistin and ceftolozane/tazobactam was supported in vitro by synergistic and bactericidal effects against OXA-23-type carbapenemase-producing CRAB isolates.

All 10 CRAB isolates in our study carried the OXA-58-type and OXA-23-type carbapenem-hydrolyzing oxacillinase. Majority of the CRAB isolates in the Republic of Korea were identified to be carrying bla $\mathrm{OXA}-23^{29}{ }^{29}$ All $10 \mathrm{CRAB}$ isolates had MIC of $64 \mathrm{mg} / \mathrm{L}$ to meropenem, and $90 \%$ of the CRAB isolates had MIC of $\leq 1$ $\mathrm{mg} / \mathrm{L}$ to tigecycline. Particularly, MICs to colistin ranged from 2 to $8 \mathrm{mg} / \mathrm{L}$. Notably, for the time-kill method, using an antibiotic concentration of $1 \times$ MIC in our study failed to show a stable bactericidal effect of antibiotic monotherapy against the 10 CRAB isolates. In this scenario, combination antibiotic therapy can become the ultimate resource for treating CRAB infections. In addition, it is predicted that the difference in susceptibilities to each antibiotic would have various effects on the antibiotic combination effect.

The colistin-ceftolozane/tazobactam combination demonstrated a synergistic effect in both the time-kill assay and the checkerboard method, while simultaneously showing a bactericidal effect in the time-kill assay. In contrast, the meropenem-tigecycline, meropenem-ceftolozane/tazobactam, and rifampin-meropenem combinations showed antagonistic effects for some CRAB isolates.

Ceftolozane/tazobactam, a novel beta-lactam/beta-lactamase inhibitor, has demonstrated potent in vitro activity against Pseudomonas aeruginosa, including carbapenem-resistant isolates, except for class B carbapenemase producers, but poor activity against $A$. baumannii isolates. ${ }^{30}$ The susceptibility of CRAB isolates to ceftolozane/tazobactam was poor with a MIC range of $16-128 \mathrm{mg} / \mathrm{L}$, which was in accordance with previous results. ${ }^{31}$ However, our findings identified the potential to induce synergistic interaction in combination with different antibiotics.

For all 10 CRAB isolates, the time-kill curves showed a significant synergistic bactericidal activity of the colistin-ceftolozane/tazobactam combination at $0.5 \times$ MIC (Figure 3). This finding has promising implications for using lower doses of colistin in treatment, thereby reducing its potential nephrotoxic effect.

In our findings, for the meropenem-tigecycline combination, in vitro synergistic activities were found in $90 \%$ and $50 \%$ of the 10 CRAB isolates in terms of the checkerboard and time-kill assays, respectively. However, in vitro antagonistic activities were found in $20 \%$ of the 10 CRAB isolates in terms of the time-kill assay. A previous meta-analysis revealed a synergistic rate of $24.5 \%$ and $20.0 \%$ for CRAB isolates using the checkerboard (36 studies) and the time-kill method (35 studies), respectively. ${ }^{19}$ Probably, the high susceptibility rate (90\%) of the CRAB isolates to tigecycline in our study may have contributed to these results. A recent clinical study reported that the tigecycline-colistin combination was associated with a higher mortality rate when the MIC of tigecycline was > $2 \mathrm{mg} / \mathrm{L}$, which was achieved with the combination of a carbapenem and colistin. ${ }^{32}$ Therefore, it is important to know how to better select from the existing antibiotic treatment regimens according to the phenotype of antibiotic resistance (hospital antibiogram) to achieve an improved clinical outcome. However, non-colistin-based combination regimens may have an important role in the treatment of CRAB infections, for those who are concerned about the nephrotoxic side effects and the emergence of hetero-resistance or resistance to colistin of CRAB isolates.

In our study, we incubated CRAB in the presence of antibiotics for $24 \mathrm{~h}$, as a result, the regrowth phenomenon was observed in the time-kill assay of the CRAB 34 isolate at $4 \mathrm{~h}$ after inoculation for the colistin-ceftolozane/tazobactam combination (both at a concentration of $1 \times$ MIC) $($ Figure 2 ). Although most of the time-kill analyses incubated bacteria in the presence of antibiotics for $24 \mathrm{~h}$, incubation for $48 \mathrm{~h}$ may be better to detect the regrowth phenomenon considering the selective amplification of the resistant subpopulation. ${ }^{33}$ A previous study reported the regrowth phenomenon commonly detected in time-kill assays using colistin, despite the in vitro antimicrobial activity of colistin against the CRAB isolates. ${ }^{16}$

In more than $50 \%$ of the 10 clinical isolates, the antibiotic combinations showing both bactericidal activity and synergistic effect at the same time-point, were colistin-ceftolozane/tazobactam and colistin-meropenem (Figure 1). In our study, the meropenem-colistin combination provided $30 \%$ and $50 \%$ synergistic activities in the checkerboard and time-kill assays, respectively. The meropenem-colistin combination remains an area of active research for the treatment of CRAB infections. Based on previous studies, a time-kill investigation of antibiotic combinations against $12 \mathrm{CRAB}$ isolates at $5 \times 10^{5} \mathrm{CFU} / \mathrm{mL}$ identified the meropenem-colistin combination as the most synergistic combination. ${ }^{34}$ Regarding synergy rates, the checkerboard and time-kill assays yielded $60-73.3 \%$ and $60-96.1 \%$ synergistic effects, respectively. ${ }^{19,35-37}$ According to a previous meta-analysis, the synergistic rates shown by time-kill methods were significantly higher than those obtained using checkerboard, which is similar to our results. ${ }^{20,38}$ Overall, there is great discordance between the checkerboard microdilution and time-kill assay with a concordance of $35 \%$ in our study. In contrast, a previous meta-analysis showed a higher synergistic rate for CRAB isolates in a combination of meropenem and colistin than a combination of imipenem and colistin. ${ }^{20,39}$

The present study has several limitations. First, the results do not apply to CRAB isolates that produce metallo-beta-lactamase. Notably, the CRAB isolates in our study are highly resistant to meropenem and may behave differently to the combinations if MICs to meropenem are lower. In addition, this study included a small number of CRAB isolates assessed in the checkerboard and time-kill assays. The MIC values of colistin in many of the CRAB isolates were remarkably high, because only several isolates were evaluated for the in vitro synergistic and bactericidal activities of antibiotic combinations. However, it is meaningful to collect isolates from patients with CRAB bacteremia in a clinical setting. Finally, we acknowledge that in vitro studies do not always lead to similar results in clinical practice; therefore, caution is required when applying these results in clinical practice. 


\section{Conclusions}

In conclusion, the present study demonstrated that the combination of colistin and ceftolozane/tazobactam may be a promising alternative to colistin for treating $C R A B$ infections. These in vitro synergy studies can provide preliminary guidance for optimal drug combination use in treating patients with $C R A B$ infections. However, the benefits of these antibiotic combinations should be validated through multicenter randomized clinical trials.

\section{Abbreviations}

Am, amikacin; An, antagonism; A/S, ampicillin/sulbactam; Az, aztreonam; B, bactericidal; C, colistin; CA-MHB, Cation-Adjusted Mueller-Hinton $\otimes$ broth; Cef, ceftazidime; Cip, ciprofloxacin; CLSI, Clinical and Laboratory Standards Institute; CRAB, carbapenem-resistant Acinetobacter baumannii; C/T, ceftolozane/tazobactam; F, female; FICl, fractional inhibitory concentration index; F, fosfomycin; I, indifference; M, male; Me, meropenem; Mi, minocycline; MIC, minimum inhibitory concentration; $\mathrm{N}$, non-bactericidal; PCR, polymerase chain reaction; P/T; piperacillin-tazobactam; R, rifampin; Syn, synergy; T, tigecycline.

\section{Declarations}

Ethics committee approval: The study protocol was approved prior to study initiation by the Institutional Review Board of Korea University Anam Hospital [No. 2020AN0157].

Funding: This research was partly supported by the Korea Health Technology R\&D Project through the Korea Health Industry Development Institute (KHIDI), funded by the Ministry of Health and Welfare, Republic of Korea (grant number: HI20C0384), and by the National Research Foundation of Korea (NRF) grant funded by the Korea Government (Ministry of Science and ICT), (No. NRF-2019R1F1A1051267). The funding source had no role in the study design, data collection, and analysis, decision to publish, or preparation of the manuscript.

Author contributions: YKY conceived, designed, and performed the study: HSY, MGL, and YGJ contributed to the experiments and the analysis of the data. HJL, CKL, and JWS contributed to the collection of clinical isolates. YKY and YGJ wrote the manuscript.

\section{Acknowledgments: None.}

\section{Conflict of interest: None}

\section{References}

1. Peleg AY, Seifert H, Paterson DL. Acinetobacter baumannii: emergence of a successful pathogen. Clin Microbiol Rev. 21, 538-582 (2008).

2. Falagas ME, Rafailidis PI. Attributable mortality of Acinetobacter baumannii: no longer a controversial issue. Crit Care. 11, 134 (2007).

3. Lemos EV, et al. Carbapenem resistance and mortality in patients with Acinetobacter baumannii infection: systematic review and meta-analysis. Clin Microbiol Infect. 20,416-423 (2014).

4. Sheng WH, et al. A multicenter study of risk factors and outcome of hospitalized patients with infections due to carbapenem-resistant Acinetobacter baumannii. Int J Infect Dis. 14,e764-769 (2014).

5. Ayats $\mathrm{J}$, et al. Epidemiological significance of cutaneous, pharyngeal, and digestive tract colonization by multiresistant Acinetobacter baumannii in ICU patients. J Hosp Infect. 37, 287-295 (1997).

6. Shamsizadeh Z, et al. Detection of antibiotic resistant Acinetobacter baumannii in various hospital environments: potential sources for transmission of Acinetobacter infections. Environ Health Prev Med. 22, 44 (2017).

7. Kim EJ, et al. Korean National Healthcare-associated Infections Surveillance System, Intensive Care Unit Module Report: Summary of Data from July 2017 through June 2018. Korean J Healthc Assoc Infection Control Prev. 24, 69-80 (2019).

8. Kim D, et al. Increasing resistance to extended-spectrum cephalosporins, fluoroquinolone, and carbapenem in gram-negative bacilli and the emergence of carbapenem non-susceptibility in Klebsiella pneumoniae: Analysis of Korean antimicrobial resistance monitoring system (KARMS) data From 2013 to 2015. Ann Lab Med. 37, 231-239 (2017).

9. Lee H, et al. Antimicrobial resistance of major clinical pathogens in South Korea, May 2016 to April 2017: first one-year report from Kor-GLASS. Euro Surveill. 23, 1800047 (2018).

10. World Health Organization. WHO publishes list of bacteria for which new antibiotics are urgently needed. 2017. Available at: https://www.who.int/news/item/27-02-2017-who-publishes-list-of-bacteria-for-which-new-antibiotics-are-urgently-needed. Accessed 28 September 2021.

11. Piperaki ET, Tzouvelekis LS, Miriagou V, Daikos GL. Carbapenem-resistant Acinetobacter baumannii: in pursuit of an effective treatment. Clin Microbiol Infect. 25, 951-957 (2019).

12. Sullivan GJ, Delgado NN, Maharjan R, Cain AK. How antibiotics work together: molecular mechanisms behind combination therapy. Curr Opin Microbiol. 57, 31-40 (2020).

13. Tan TY, et al. In vitro antibiotic synergy in extensively drug-resistant Acinetobacter baumannii: the effect of testing by time-kill, checkerboard, and Etest methods. Antimicrob Agents Chemother. 55,436-438 (2011).

14. Soudeiha MAH, Dahdouh EA, Azar E, Sarkis DK, Daoud Z. In vitro evaluation of the colistin-carbapenem combination in clinical isolates of A. baumannii using the checkerboard, Etest, and time-kill curve techniques. Front Cell Infect Microbiol. 7, 209 (2017). 
15. Sopirala MM, et al. Synergy testing by Etest, microdilution checkerboard, and time-kill methods for pan-drug-resistant Acinetobacter baumannii. Antimicrob Agents Chemother. 54, 4678-83 (2010).

16. Ni W, et al. In vitro synergy of polymyxins with other antibiotics for Acinetobacter baumannii: a systematic review and meta-analysis. Int $J$ Antimicrob Agents. 45, 8-18 (2015).

17. Li J, Yang X, Chen L, Duan X, Jiang Z. In vitro activity of various antibiotics in combination with tigecycline against Acinetobacter baumannii: A systematic review and meta-analysis. Microb Drug Resist. 23, 982-993 (2017).

18. March GA, Bratos MA. A meta-analysis of in vitro antibiotic synergy against Acinetobacter baumannii. J Microbiol Methods. 119, 31-36 (2015).

19. Jiang Z, He X, Li J. Synergy effect of meropenem-based combinations against Acinetobacter baumannii: a systematic review and meta-analysis. Infect Drug Resist. 11, 1083-1095 (2018).

20. Zusman 0, et al. Systematic review and meta-analysis of in vitro synergy of polymyxins and carbapenems. Antimicrob Agents Chemother, 57, 5104-5111 (2013).

21. Clinical and Laboratory Standards Institute (CLSI). Performance Standards for Antimicrobial Susceptibility Testing; 26th Edn. CLSI supplement M100S. Wayne, PA, USA. 2016.

22. Clinical and Laboratory Standards Institute (CLSI). Methods for dilution antimicrobial susceptibility tests for bacteria that grow aerobically, M07-11th edition, Melvin P. Weinstein, MD: CLSI; 2018.

23. Wyeth Pharmaceuticals. Tygacil (tigecycline) for injection [Package insert]. Wyeth Pharmaceuticals Inc., Philadelphia, PA. 2005.

24. Gales AC, Reis AO, Jones RN. Contemporary assessment of antimicrobial susceptibility testing methods for polymyxin B and colistin: review of available interpretative criteria and quality control guidelines. J Clin Microbiol. 39, 183-190 (2001).

25. Hogg GM, Barr JG, Webb CH. In-vitro activity of the combination of colistin and rifampicin against multidrug-resistant strains of Acinetobacter baumannii. $J$ Antimicrob Chemother. 41, 494-495 (1998).

26. Tan TY, Ng LS, Tan E, Huang G. In vitro effect of minocycline and colistin combinations on imipenem-resistant Acinetobacter baumannii clinical isolates. J Antimicrob Chemother. 60, 421-423 (2001).

27. Principe $L$, et al. In vitro activity of doripenem in combination with various antimicrobials against multidrug-resistant Acinetobacter baumannii: possible options for the treatment of complicated infection. Microb Drug Resist. 19, 407-414 (2013).

28. Park GC, et al. In vitro interactions of antibiotic combinations of colistin, tigecycline, and doripenem against extensively drug-resistant and multidrugresistant Acinetobacter baumannii. Ann Lab Med. 36, 124-130 (2016).

29. Jun SH, et al. Clonal change of carbapenem-resistant Acinetobacter baumannii isolates in a Korean hospital. Infect Genet Evol. 93 , 104935 (2021).

30. van Duin D, Bonomo RA. Ceftazidime/avibactam and ceftolozane/tazobactam: second-generation $\beta$-lactam/ $\beta$-lactamase inhibitor combinations. Clin Infect Dis. 63, 234-241 (2016).

31. Hsueh SC, et al. In vitro activities of cefiderocol, ceftolozane/tazobactam, ceftazidime/avibactam and other comparative drugs against imipenemresistant Pseudomonas aeruginosa and Acinetobacter baumannii, and Stenotrophomonas maltophilia, all associated with bloodstream infections in Taiwan. J Antimicrob Chemother. 74, 380-386 (2019).

32. Garnacho-Montero J, et al. Acinetobacter baumannii in critically ill patients: molecular epidemiology, clinical features and predictors of mortality. Enferm Infecc Microbiol Clin. 34, 551-558 (2016).

33. Tam VH, Schilling AN, Nikolaou M. Modelling time-kill studies to discern the pharmacodynamics of meropenem. J Antimicrob Chemother. 55, 699-706 (2015).

34. Sheng WH, et al. Comparative in vitro antimicrobial susceptibilities and synergistic activities of antimicrobial combinations against carbapenem-resistant Acinetobacter species: Acinetobacter baumannii versus Acinetobacter genospecies 3 and 13TU. Diagn Microbiol Infect Dis 70, 380-386 (2011).

35. Timurkaynak F, et al. In vitro activities of non-traditional antimicrobials alone or in combination against multidrug-resistant strains of Pseudomonas aeruginosa and Acinetobacter baumannii isolated from intensive care units. Int J Antimicrob Agents. 27, 224-228 (2006).

36. Pongpech $\mathrm{P}$, et al. Antibacterial activity of carbapenem-based combinations against multidrug-resistant Acinetobacter baumannii. J Med Assoc Thai. 93 , 161-171 (2010).

37. Pankuch GA, Lin G, Seifert H, Appelbaum PC. Activity of meropenem with and without ciprofloxacin and colistin against Pseudomonas aeruginosa and Acinetobacter baumannii. Antimicrob Agents Chemother. 52, 333-336 (2008).

38. March GA, Bratos MA. A meta-analysis of in vitro antibiotic synergy against Acinetobacter baumannii. J Microbiol Methods. 119, 31-36 (2015).

39. Soudeiha MAH, Dahdouh EA, Azar E, Sarkis DK, Daoud Z. In vitro evaluation of the colistin-carbapenem combination in clinical isolates of A. baumannii using the checkerboard, Etest, and time-kill curve techniques. Front Cell Infect Microbiol. 7, 209 (2017).

\section{Tables}

Table 1. Polymerase chain reaction (PCR) primers, conditions, and product size for the detection of carbapenemases among Acinetobacter baumannii strains 


\begin{tabular}{|c|c|c|c|}
\hline Target gene & Primers (5'à $\left.3^{\prime}\right)$ & Annealing temperature for multiplex PCR & Product size (bp) \\
\hline \multirow[t]{2}{*}{$b / a_{\mid \mathrm{MP}-1}$} & ACCGCAGCAGAGTCTTTGCC & $55^{\circ} \mathrm{C}$ & 587 \\
\hline & ACAACCAGTTTTGCCTTACC & & \\
\hline \multirow[t]{2}{*}{$b / a_{\mid \mathrm{MP}-2}$} & GTTTTATGTGTATGCTTCC & $55^{\circ} \mathrm{C}$ & 678 \\
\hline & AGCCTGTTCCCATGTAC & & \\
\hline \multirow[t]{2}{*}{$b / a_{\mathrm{VIM}-1}$} & GGGAGCCGAGTGGTGAGT & $55^{\circ} \mathrm{C}$ & 519 \\
\hline & GGCACAACCACCGTATAG & & \\
\hline \multirow[t]{2}{*}{$b / a_{\mathrm{VIM}-2}$} & ATGTTCAAACTTTTGAGTAAG & $55^{\circ} \mathrm{C}$ & 801 \\
\hline & CTACTCAACGACTGAGCG & & \\
\hline \multirow[t]{2}{*}{$b / a_{\text {oxa-23 }}$} & GATCGGATTGGAGAACCAGA & $52^{\circ} \mathrm{C}$ & 501 \\
\hline & ATTTCTGACCGCATTTCCAT & & \\
\hline \multirow[t]{2}{*}{$b / a_{\text {oxa-24 }}$} & GGTTAGTTGGCCCCCTTAAA & $52{ }^{\circ} \mathrm{C}$ & 246 \\
\hline & AGTTGAGCGAAAAGGGGATT & & \\
\hline \multirow[t]{2}{*}{$b / a_{\text {oxa-51 }}$} & TAATGCTTTGATCGGCCTTG & $52^{\circ} \mathrm{C}$ & 353 \\
\hline & TGGATTGCACTTCATCTTGG & & \\
\hline \multirow[t]{2}{*}{$b / a_{\text {oxa- }-58}$} & AAGTATTGGGGCTTGTGCTG & $52{ }^{\circ} \mathrm{C}$ & 599 \\
\hline & ССССТCTGCGCTCTACATAC & & \\
\hline
\end{tabular}

Table 2. Clinical and microbiological characteristics of Acinetobacter baumannii isolates

\begin{tabular}{|c|c|c|c|c|c|c|c|c|c|c|c|c|c|c|c|c|c|c|c|}
\hline \multirow[t]{2}{*}{ Isolate no. } & \multicolumn{4}{|c|}{ Patient information } & \multicolumn{2}{|c|}{$\begin{array}{c}\text { Carbapenemase } \\
\text { genes }\end{array}$} & \multicolumn{13}{|c|}{ MICs of antimicrobial agents $(\mathrm{mg} / \mathrm{L})^{*}$} \\
\hline & Age & Sex & $\begin{array}{l}\text { Clinical } \\
\text { specimen }\end{array}$ & $\begin{array}{l}\text { In-hospital } \\
\text { mortality }\end{array}$ & $b l a_{0 \times a-51}$ & $b l a_{0 \times a-23}$ & Me & $\mathrm{C}$ & $\mathbf{T}$ & $\begin{array}{l}\mathrm{C} / \\
\mathbf{T}\end{array}$ & $\mathbf{R}$ & Cip & $\mathbf{F}$ & Mi & Az & Cef & $\mathbf{A} / \mathbf{S}$ & $\mathbf{P} / \mathbf{T}$ & Am \\
\hline $\begin{array}{l}\text { ATCC2785 } \\
3\end{array}$ & - & - & - & - & - & - & 0.25 & 2 & 4 & 0.5 & 64 & $\begin{array}{c}0.12 \\
5\end{array}$ & 4 & 16 & 8 & 2 & $>128$ & 4 & 1 \\
\hline CRAB 19 & 90 & $\mathrm{M}$ & Blood & No & + & + & 64 & 2 & 0.5 & 128 & 4 & 16 & $>128$ & 0.25 & 128 & $>128$ & 64 & $>128$ & 128 \\
\hline CRAB 32 & 66 & $F$ & Blood & Yes & + & + & 64 & 8 & 0.5 & 16 & 4 & 32 & 128 & 0.25 & 64 & 128 & 64 & $>128$ & $>128$ \\
\hline CRAB 33 & 95 & $F$ & Blood & Yes & + & + & 64 & 8 & 0.5 & 32 & 4 & 64 & 128 & 0.25 & 64 & 128 & 64 & $>128$ & $>128$ \\
\hline CRAB 34 & 81 & $\mathrm{~F}$ & Blood & No & + & + & 64 & 8 & 0.5 & 64 & $>128$ & 64 & 128 & 0.25 & 128 & $>128$ & 64 & $>128$ & $>128$ \\
\hline CRAB 35 & 75 & $\mathrm{~F}$ & Blood & No & + & + & 64 & 4 & 0.5 & 64 & $>128$ & 64 & 128 & 0.25 & 128 & $>128$ & 64 & $>128$ & $>128$ \\
\hline CRAB 36 & 57 & $F$ & Blood & No & + & + & 64 & 8 & 0.5 & 64 & $>128$ & 128 & 128 & 0.25 & 128 & $>128$ & 64 & $>128$ & $>128$ \\
\hline CRAB 37 & 66 & $\mathrm{M}$ & Blood & No & + & + & 64 & 8 & 0.5 & 32 & $>128$ & 64 & 128 & 0.25 & 128 & $>128$ & 64 & $>128$ & $>128$ \\
\hline CRAB 38 & 81 & $F$ & Blood & Yes & + & + & 64 & 8 & 0.5 & 64 & $>128$ & 64 & 128 & 0.25 & 128 & $>128$ & 64 & $>128$ & $>128$ \\
\hline CRAB 39 & 68 & $\mathrm{M}$ & Blood & Yes & + & + & 64 & 4 & 1 & 32 & $>128$ & 64 & 128 & 0.25 & 128 & $>128$ & 64 & $>128$ & $>128$ \\
\hline CRAB 40 & 89 & $\mathrm{M}$ & Blood & Yes & + & + & 64 & 8 & 4 & 32 & $>128$ & 128 & 64 & 0.5 & 64 & $>128$ & 64 & $>128$ & $>128$ \\
\hline
\end{tabular}

Am, amikacin; A/S, ampicillin/sulbactam; Az, aztreonam; Cef, ceftazidime; CRAB, carbapenem-resistant Acinetobacter baumannii; Cip, ciprofloxacin; C, colistin; $\mathrm{C} / \mathrm{T}$, ceftolozane/tazobactam; $\mathrm{F}$, female; F, fosfomycin; $\mathrm{M}$, male; Me, meropenem; Mi, minocycline; MIC, minimum inhibitory concentration; $\mathrm{P} / \mathrm{T}$; piperacillintazobactam; R, rifampin; T, tigecycline

*Gray cell, non-susceptible; white cell, susceptible

Table 3. Results of checkerboard assay for two-drug combinations against clinical isolates of Acinetobacter baumannii. 


\begin{tabular}{|c|c|c|c|c|c|c|c|}
\hline & \multirow[t]{2}{*}{$\begin{array}{l}\text { Clinical } \\
\text { isolates }\end{array}$} & \multicolumn{2}{|c|}{$\begin{array}{l}\text { MIC of a single agent } \\
(\mathrm{mg} / \mathrm{L})\end{array}$} & \multicolumn{2}{|c|}{$\begin{array}{l}\text { MIC in combination } \\
(\mathrm{mg} / \mathrm{L})\end{array}$} & \multirow[t]{2}{*}{$\mathrm{FICl}$} & \multirow[t]{2}{*}{$\begin{array}{l}\text { Result of checkerboard } \\
\text { assay }\end{array}$} \\
\hline & & Drug A & Drug B & Drug A & Drug B & & \\
\hline \multirow[t]{10}{*}{ Meropenem (A) + colistin (B) } & CRAB 19 & 64 & 2 & 4 & 0.25 & 0.19 & Synergistic \\
\hline & CRAB 32 & 64 & 8 & 16 & 0.5 & 0.31 & Synergistic \\
\hline & CRAB 33 & 64 & 8 & 0.125 & 0.25 & 0.03 & Synergistic \\
\hline & CRAB 34 & 64 & 8 & 32 & 0.25 & 0.53 & Additive \\
\hline & CRAB 35 & 64 & 8 & 32 & 0.25 & 0.56 & Additive \\
\hline & CRAB 36 & 64 & 8 & 32 & 0.5 & 0.56 & Additive \\
\hline & CRAB 37 & 64 & 8 & 32 & 0.25 & 0.53 & Additive \\
\hline & CRAB 38 & 64 & 8 & 32 & 0.25 & 0.53 & Additive \\
\hline & CRAB 39 & 64 & 4 & 32 & 0.25 & 0.56 & Additive \\
\hline & CRAB 40 & 64 & 8 & 32 & 0.25 & 0.53 & Additive \\
\hline \multirow[t]{10}{*}{ Meropenem (A) + tigecycline (B) } & CRAB 19 & 64 & 0.5 & 0.125 & 0.03 & 0.06 & Synergistic \\
\hline & CRAB 32 & 64 & 0.5 & 8 & 0.125 & 0.38 & Synergistic \\
\hline & CRAB 33 & 64 & 0.5 & 32 & 0.5 & 1.5 & Indifferent \\
\hline & CRAB 34 & 64 & 0.5 & 16 & 0.125 & 0.5 & Synergistic \\
\hline & CRAB 35 & 64 & 0.5 & 16 & 0.125 & 0.5 & Synergistic \\
\hline & CRAB 36 & 64 & 0.5 & 0.125 & 0.125 & 0.25 & Synergistic \\
\hline & CRAB 37 & 64 & 0.5 & 0.125 & 0.125 & 0.25 & Synergistic \\
\hline & CRAB 38 & 64 & 0.5 & 16 & 0.125 & 0.5 & Synergistic \\
\hline & CRAB 39 & 64 & 1 & 16 & 0.125 & 0.38 & Synergistic \\
\hline & CRAB 40 & 64 & 4 & 8 & 0.125 & 0.16 & Synergistic \\
\hline \multirow[t]{10}{*}{ Meropenem (A) + rifampin (B) } & CRAB 19 & 64 & 4 & 32 & 0.25 & 0.56 & Additive \\
\hline & CRAB 32 & 64 & 4 & 32 & 4 & 1.5 & Indifferent \\
\hline & CRAB 33 & 64 & 4 & 16 & 2 & 0.75 & Additive \\
\hline & CRAB 34 & 64 & 256 & 4 & 8 & 0.09 & Synergistic \\
\hline & CRAB 35 & 64 & 256 & 16 & 4 & 0.27 & Synergistic \\
\hline & CRAB 36 & 64 & 256 & 16 & 8 & 0.28 & Synergistic \\
\hline & CRAB 37 & 64 & 256 & 16 & 4 & 0.27 & Synergistic \\
\hline & CRAB 38 & 64 & 256 & 16 & 4 & 0.27 & Synergistic \\
\hline & CRAB 39 & 64 & 256 & 16 & 4 & 0.27 & Synergistic \\
\hline & CRAB 40 & 64 & 256 & 8 & 8 & 0.16 & Synergistic \\
\hline \multirow{10}{*}{$\begin{array}{l}\text { Meropenem }(\mathrm{A})+ \\
\text { ceftolozane/tazobactam (B) }\end{array}$} & CRAB 19 & 64 & 128 & 32 & 16 & 0.63 & Additive \\
\hline & CRAB 32 & 64 & 16 & 8 & 4 & 0.38 & Synergistic \\
\hline & CRAB 33 & 64 & 32 & 8 & 16 & 0.63 & Additive \\
\hline & CRAB 34 & 64 & 64 & 8 & 4 & 0.19 & Synergistic \\
\hline & CRAB 35 & 64 & 64 & 2 & 2 & 0.06 & Synergistic \\
\hline & CRAB 36 & 64 & 64 & 32 & 32 & 1 & Additive \\
\hline & CRAB 37 & 64 & 32 & 4 & 2 & 0.13 & Synergistic \\
\hline & CRAB 38 & 64 & 64 & 16 & 2 & 0.28 & Synergistic \\
\hline & CRAB 39 & 64 & 32 & 2 & 4 & 0.16 & Synergistic \\
\hline & CRAB 40 & 64 & 32 & 8 & 8 & 0.38 & Synergistic \\
\hline Colistin (A) + tigecycline (B) & CRAB 19 & 2 & 0.5 & 0.006 & 0.03 & 0.06 & Synergistic \\
\hline
\end{tabular}




\begin{tabular}{|c|c|c|c|c|c|c|c|}
\hline & CRAB 32 & 8 & 0.5 & 4 & 0.25 & 1 & Additive \\
\hline & CRAB 33 & 8 & 0.5 & 0.5 & 0.25 & 0.56 & Additive \\
\hline & CRAB 34 & 8 & 0.5 & 8 & 0.5 & 2 & Indifferent \\
\hline & CRAB 35 & 8 & 0.5 & 4 & 0.25 & 1.5 & Indifferent \\
\hline & CRAB 36 & 8 & 0.5 & 2 & 0.125 & 0.5 & Synergistic \\
\hline & CRAB 37 & 8 & 0.5 & 8 & 0.5 & 2 & Indifferent \\
\hline & CRAB 38 & 8 & 0.5 & 8 & 0.5 & 2 & Indifferent \\
\hline & CRAB 39 & 4 & 1 & 4 & 1 & 2 & Indifferent \\
\hline & CRAB 40 & 8 & 4 & 0.125 & 1 & 0.27 & Synergistic \\
\hline \multirow[t]{10}{*}{ Colistin (A) + ceftolozane/tazobactam (B) } & CRAB 19 & 2 & 128 & 2 & 128 & 2 & Indifferent \\
\hline & CRAB 32 & 8 & 16 & 4 & 8 & 1 & Additive \\
\hline & CRAB 33 & 8 & 32 & 0.125 & 2 & 0.08 & Synergistic \\
\hline & CRAB 34 & 8 & 64 & 0.5 & 8 & 0.19 & Synergistic \\
\hline & CRAB 35 & 8 & 64 & 0.25 & 16 & 0.31 & Synergistic \\
\hline & CRAB 36 & 8 & 64 & 8 & 64 & 2 & Indifferent \\
\hline & CRAB 37 & 8 & 32 & 1 & 16 & 0.63 & Additive \\
\hline & CRAB 38 & 8 & 64 & 2 & 2 & 0.28 & Synergistic \\
\hline & CRAB 39 & 4 & 32 & 0.25 & 4 & 0.19 & Synergistic \\
\hline & CRAB 40 & 8 & 32 & 0.125 & 8 & 0.27 & Synergistic \\
\hline
\end{tabular}

CRAB, carbapenem-resistant Acinetobacter baumannir; FICl, fractional inhibitory concentration index; MIC, minimum inhibitory concentration

Table 4. Results of time-kill assay and bactericidal activity of two-drug combinations $(1 \times \mathrm{MIC})$ against each Acinetobacter baumannii isolate

\begin{tabular}{|c|c|c|c|c|c|c|c|c|c|c|c|c|}
\hline \multirow[t]{2}{*}{ Isolate } & \multicolumn{6}{|c|}{ Time-kill assay } & \multicolumn{6}{|c|}{$\begin{array}{l}\text { Bactericidal activity } \\
\text { (Timepoint, hours; } 2 / 4 / 6 / 8 / 12 / 24 \text { ) }\end{array}$} \\
\hline & $\mathrm{Me}+\mathrm{T}$ & $\mathrm{Me}+\mathrm{C}$ & $\mathrm{Me}+\mathrm{R}$ & $\mathrm{Me}+\mathrm{C} / \mathrm{T}$ & $\mathrm{C}+\mathrm{T}$ & $\mathrm{C}+\mathrm{C} / \mathrm{T}$ & $\mathrm{Me}+\mathrm{T}$ & $\mathrm{Me}+\mathrm{C}$ & $\mathrm{Me}+\mathrm{R}$ & $\mathrm{Me}+\mathrm{C} / \mathrm{T}$ & $\mathrm{C}+\mathrm{T}$ & $\mathrm{C}+\mathrm{C} / \mathrm{T}$ \\
\hline $\begin{array}{l}\text { CRAB } \\
19\end{array}$ & Syn. & Syn. & Syn. & Syn. & Syn. & Syn. & N/N/N/N/B & N/N/B/B/B & N/N/B/B/B & N/N/N/B/B & N/B/B/B/B & N/N/B/B/B \\
\hline $\begin{array}{l}\text { CRAB } \\
32\end{array}$ & Syn. & Syn. & $\mathrm{I}$. & Syn. & Syn. & Syn. & N/N/N/B/B & N/N/B/B/B & $\mathrm{N} / \mathrm{N} / \mathrm{N} / \mathrm{N} / \mathrm{N}$ & N/N/N/N/B & N/N/N/B/B & N/N/B/B/B \\
\hline $\begin{array}{l}\text { CRAB } \\
33\end{array}$ & Syn. & Syn. & I. & Syn. & Syn. & Syn. & $\mathrm{N} / \mathrm{N} / \mathrm{N} / \mathrm{N} / \mathrm{N}$ & N/N/N/B/B & $\mathrm{N} / \mathrm{N} / \mathrm{N} / \mathrm{N} / \mathrm{N}$ & $\mathrm{N} / \mathrm{N} / \mathrm{N} / \mathrm{N} / \mathrm{N}$ & N/N/N/N/B & N/N/N/B/B \\
\hline $\begin{array}{l}\text { CRAB } \\
34\end{array}$ & Syn. & Syn. & Syn. & Syn. & Syn. & Syn. & N/N/N/N/B & N/N/N/B/B & N/N/N/B/B & N/N/N/N/B & N/B/B/B/B & B/N/B/B/B \\
\hline $\begin{array}{l}\text { CRAB } \\
35\end{array}$ & Syn. & Syn. & $\mathrm{I}$. & $\mathrm{I}$. & Syn. & Syn. & N/N/N/N/N & N/N/N/B/B & $\mathrm{N} / \mathrm{N} / \mathrm{N} / \mathrm{N} / \mathrm{N}$ & N/N/N/N/N & N/N/N/N/B & N/N/N/B/B \\
\hline $\begin{array}{l}\text { CRAB } \\
36\end{array}$ & Syn. & Syn. & $\mathrm{I}$. & Syn. & Syn. & Syn. & N/N/N/B/B & N/N/N/B/B & $\mathrm{N} / \mathrm{N} / \mathrm{N} / \mathrm{N} / \mathrm{N}$ & N/N/N/N/B & N/N/B/B/B & N/N/N/B/B \\
\hline $\begin{array}{l}\text { CRAB } \\
37\end{array}$ & Syn. & Syn. & Syn. & Syn. & Syn. & Syn. & N/N/N/B/B & N/N/N/B/B & N/N/N/B/B & N/N/N/N/B & N/B/B/B/B & N/N/N/B/B \\
\hline $\begin{array}{l}\text { CRAB } \\
38\end{array}$ & $\mathrm{I}$. & Syn. & An. & I. & Syn. & Syn. & $\mathrm{N} / \mathrm{N} / \mathrm{N} / \mathrm{N} / \mathrm{N}$ & N/N/N/B/B & $\mathrm{N} / \mathrm{N} / \mathrm{N} / \mathrm{N} / \mathrm{N}$ & N/N/N/N/N & N/N/N/N/B & N/N/N/B/B \\
\hline $\begin{array}{l}\text { CRAB } \\
39\end{array}$ & An. & Syn. & An. & $\mathrm{I}$. & Syn. & Syn. & $\mathrm{N} / \mathrm{N} / \mathrm{N} / \mathrm{N} / \mathrm{N}$ & N/N/N/B/B & $\mathrm{N} / \mathrm{N} / \mathrm{N} / \mathrm{N} / \mathrm{N}$ & $\mathrm{N} / \mathrm{N} / \mathrm{N} / \mathrm{N} / \mathrm{N}$ & N/N/N/B/B & N/N/N/B/B \\
\hline $\begin{array}{l}\text { CRAB } \\
40\end{array}$ & $\mathrm{I}$. & Syn. & An. & An. & Syn. & Syn. & N/N/N/N/N & N/N/N/B/B & $\mathrm{N} / \mathrm{N} / \mathrm{N} / \mathrm{N} / \mathrm{N}$ & $\mathrm{N} / \mathrm{N} / \mathrm{N} / \mathrm{N} / \mathrm{N}$ & N/N/N/B/B & N/N/N/B/B \\
\hline
\end{tabular}

An, antagonism; B, bactericidal; C, colistin; C/T, ceftolozane/tazobactam; I, indifference; Me, meropenem; N, non-bactericidal; R, rifampin; Syn, synergy; T, tigecycline 
Table 5. Comparison of checkerboard assay and time-kill assay associated with the bactericidal activity of two-drug combinations ( $1 \times$ MIC) against each Acinetobacter baumannii isolate

\begin{tabular}{|c|c|c|c|c|c|c|c|c|c|c|c|c|}
\hline \multirow[t]{2}{*}{ Isolate } & \multicolumn{6}{|c|}{ Checkerboard assay results } & \multicolumn{6}{|c|}{ Time-kill assay results } \\
\hline & $\mathbf{M}+\mathbf{T}$ & $\mathbf{M}+\mathbf{C}$ & $\mathbf{M}+\mathbf{R}$ & $\mathbf{M}+\mathbf{C} / \mathbf{T}$ & $\mathbf{C}+\mathbf{T}$ & $\mathbf{C}+\mathrm{C} / \mathrm{T}$ & $\mathbf{M}+\mathbf{T}$ & $\mathbf{M}+\mathbf{C}$ & $\mathbf{M}+\mathbf{R}$ & $\mathbf{M}+\mathbf{C} / \mathbf{T}$ & $\mathbf{C}+\mathbf{T}$ & $\mathbf{C}+\mathrm{C} / \mathrm{T}$ \\
\hline CRAB 19 & Syn. & Syn. & Additive & Additive & Syn. & I. & I. $/ \mathrm{B}$. & Syn./B. & Syn./B. & Syn./B. & Syn./B. & Syn./B. \\
\hline CRAB 32 & Syn. & Syn. & I. & Syn. & Additive & Additive & I./B. & Syn./B. & $\mathrm{I} / \mathrm{N}$. & Syn./B. & I. $/ B$. & Syn./B. \\
\hline CRAB 33 & I. & Syn. & Additive & Additive & Additive & Syn. & Syn./N. & Syn./B. & I. $/ \mathrm{N}$. & Syn./N. & Syn./B. & Syn./B. \\
\hline CRAB 34 & Syn. & Additive & Syn. & Syn. & I. & Syn. & Syn./B. & I. $/ B$. & Syn./B. & Syn./B. & Syn./B. & Syn./B. \\
\hline CRAB 35 & Syn. & Additive & Syn. & Syn. & I. & Syn. & Syn./N. & Syn./B. & I. $/ \mathrm{N}$. & $\mathrm{I} / \mathrm{N}$. & Syn./B. & Syn./B. \\
\hline CRAB 36 & Syn. & Additive & Syn. & Additive & Syn. & I. & Syn./B. & I. $/ B$. & I. $/ \mathrm{N}$. & Syn./B. & Syn./B. & I.B. \\
\hline CRAB 37 & Syn. & Additive & Syn. & Syn. & I. & Additive & Syn./B. & Syn./B. & Syn./B. & Syn./B. & Syn./B. & Syn./B. \\
\hline CRAB 38 & Syn. & Additive & Syn. & Syn. & I. & Syn. & $\mathrm{An} / \mathrm{N}$. & I. $/ B$. & $\mathrm{An} / \mathrm{N}$. & $\mathrm{An} / \mathrm{B}$. & I. $/ B$. & I. $/ \mathrm{B}$. \\
\hline CRAB 39 & Syn. & Additive & Syn. & Syn. & I. & Syn. & $\mathrm{An} / \mathrm{N}$. & I. $/ B$. & $\mathrm{An} / \mathrm{N}$. & $\mathrm{An} / \mathrm{N}$. & Syn./B. & Syn./B. \\
\hline CRAB 40 & Syn. & Additive & Syn. & Syn. & Syn. & Syn. & $\mathrm{I} / \mathrm{N}$. & I./B. & An. N. & An. $/ \mathrm{N}$. & I. B. & I./B. \\
\hline
\end{tabular}

An, antagonism; B, bactericidal; C, colistin; C/T, ceftolozane/tazobactam; I. indifference; M, meropenem; N, non-bactericidal; R, rifampin; Syn, synergy; T, tigecycline

*Gray cells mean that the results of the time-kill assay were consistent with the results of the checkerboard assays.

\section{Figures}

\section{Figure 1}

Results of time-kill assay and bactericidal activity of two-drug combinations against 10 Acinetobacter baumannii isolates. The percentage in the figure indicates the ratio of isolates showing bactericidal or synergistic effect among all 10 CRAB isolates.

C, colistin; C/T, ceftolozane/tazobactam; Me, meropenem; R, rifampin; T, tigecycline

Figure 2

Time-kill curves of $A$. baumannii isolates (CRAB 34) at $1 \times$ MIC concentration for six combinations of antibiotics

Figure 3

Time-kill curves of the $10 \mathrm{~A}$. baumannii isolates for six combinations of antibiotics with the different concentrations

C, colistin; C/T, ceftolozane/tazobactam; Me, meropenem; R, rifampin; T, tigecycline

\section{Supplementary Files}

This is a list of supplementary files associated with this preprint. Click to download.

- SupplementaryfileYoonetal.xlsx 